\title{
Justicia procedimental y aceptación al tratamiento psicológico de los adolescentes que infringieron la ley penal
}

\section{Procedural justice and responsiveness to psychological treatment of juvenile delinquents}

\author{
Amelia Eréndida López Escobar \\ Martha Frías Armenta \\ Universidad de Sonora
}

\begin{abstract}
Resumen
El propósito del presente trabajo fue probar un modelo estructural para analizar la relación entre justicia procedimental y la aceptación del tratamiento psicológico de los adolescentes en conflicto con la ley. La muestra estuvo conformada por 92 jóvenes, que se encontraban estaban recibiendo tratamiento en internamiento o en externamiento por haber sido sentenciados por el juez de menores. Los participantes contestaron un cuestionario con reactivos acerca de la percepción del procedimiento judicial, el trato de la policía, la aceptación de la sentencia y el tratamiento psicológico que recibían. Los resultados revelaron que la percepción del adolescente en relación al trato del juez predijo de manera positiva la aceptación al tratamiento psicológico y judicial.

Palabras clave: Justicia procedimental, tratamiento psicológico, adolecentes, sentencia, percepción.
\end{abstract}

Nota del autor

Amelia Eréndida López Escobar, Departamento de Psicología, Universidad de Sonora; Martha Frías Armenta, Departamento de Psicología, Universidad de Sonora.

La correspondencia en relación con este artículo debe dirigirse a Martha Frías Armenta, Departamento de Psicología, Universidad de Sonora, Hermosillo, Sonora, 83000.

Dirección electrónica: marthafrias@sociales.uson.mx 


\begin{abstract}
The aim of this research is to prove a structural model to analyze the relationship between procedural justice and the responsiveness to psychological treatment in juvenile delinquents. The sample were 92 adolescents who were sentenced by a juvenile court. Participants answered a questionnaire related to perception of the judicial procedure, treatment of the police, acceptation of the sentence and psychological treatment that received. Results revealed that of the adolescent positive perception of judge predicted the responsiveness of psychological treatment.

Keywords: procedural justice, sentence, perception, responsiveness, treatment, juvenile delinquents.
\end{abstract}

En los últimos años en México (Jiménez, 2011), el porcentaje de delitos cometidos por menores de 8 a 17 años y jóvenes de 18 a 29 años ha aumentado de forma gradual. Particularmente, en el Estado de Sonora, donde las estadísticas muestran un aumento paulatino en los ingresos a los centros de internamiento de menores que cometen un delito, para 2008 fue un total de 1,348 adolescentes y para 2009 aumentó a 1,571 jóvenes en conflicto con la ley (INEGI, 2011). Las estadísticas de la República Mexicana desplegadas por el Instituto Nacional de Estadística, Geografía e Informática (INEGI, 2010) describen los datos del 2004 sobre los menores infractores en el interior del país y reportan a 45,593 adolescentes en conflicto con la ley. La entidad con mayor número de menores infractores registrados es Baja California con 12,542 y por su parte, Tlaxcala se ubica como el estado de menor incidencia con 90 casos, mientras que a Sonora le corresponde un octavo lugar con 1,604 adolescentes puestos a disposición de las instituciones jurídicas.

En México, se modificó el Artículo 18 Constitucional en diciembre de 2005 (Diario, Oficial de la Federación, 2005) y las leyes estatales de menores se tuvieron que transformar en el mismo sentido. La reforma proclama un sistema integral de justicia para adolescentes, entre los doce años cumplidos y menos de 18, que hayan infringido las leyes penales, además, indica que se deberán garantizar a los adolescentes los derechos fundamentales estipulados en la Constitución. Para los menores de 12 años, se estipula la asistencia social y las medidas serán la orientación, la protección y el tratamiento, evitando en todo momento la privación de la libertad. Para los mayores de 14 años, el encarcelamiento se establece como medida extrema, así mismo, se asegurarán a los adolescentes las garantías de debido proceso. La protección integral procura la reintegración familiar y social del menor y el pleno desarrollo de su persona y capacidades. Los procedimientos administrativos fueron eliminados y se establecieron tribunales especializados para menores. Adicionalmente, la reforma estatuye las formas alternativas de justicia, como la restaurativa, la mediación y la conciliación (Constitución Política Mexicana, 2013). La política pública radica en la búsqueda del bienestar del menor con una intervención 
judicial mínima. La protección de los derechos fundamentales de los menores es primordial, preponderantemente la presunción de inocencia, el derecho a no responder la acusación, a la defensa adecuada, a la presencia de los padres, a la confrontación y a la apelación.

Estas transformaciones legales y de políticas públicas han modificado el tratamiento hacia los menores, sin embargo, el involucramiento de los jóvenes en la delincuencia sigue en aumento. Por lo tanto, es importante esclarecer cuáles son las fallas del sistema que no permiten la prevención y la disminución de la delincuencia. En este sentido, se ha estudiado, en el campo de la psicología jurídica, la justicia procedimental que consiste en el sentimiento de equidad o imparcialidad percibidos por un individuo que participa en un proceso judicial. La teoría nos indica que esta percepción positiva sobre el proceso produce resultados más positivos en los individuos y pudiera incidir en la disminución de la conducta delictiva posterior (Tyler \& Blader, 2000). El estudio de la relación entre justicia procedimental y aceptación del tratamiento pudiera elucidar algunas de las fallas del sistema de justicia juvenil y proporcionar las pautas para ayudar a reducir las reincidencias de los adolescentes en conflicto con la ley.

Existen muy pocos estudios a nivel internacional que consideren los efectos de la justicia procedimental en los jóvenes. Igualmente, es poca la investigación que analiza si las mismas variables que se toman en cuenta para los adultos en la percepción de la justicia procedimental serán aplicables para los jóvenes y los niños. Existen interrogantes acerca de si los jóvenes valoran la justicia procedimental igual que los adultos; o si las experiencias de aquéllos con el sistema legal impactan en su percepción de justicia y de respeto al sistema.

En México, no existen investigaciones sobre justicia procedimental. Esta es una de las primeras en analizar los efectos de la percepción de justicia y la adherencia del tratamiento en los jóvenes.

\section{Justicia}

La noción de justicia varía dependiendo de las épocas y culturas, sin embargo, el término alude a la idea de cierta igualdad (Perelman, 1964). Desde el punto de vista formal es un conjunto de reglas que se establecen para la interacción de los individuos en la sociedad. La justicia es considerada como una cualidad de una organización social y está relacionada con el desarrollo personal en un contexto social, cada individuo deberá de recibir un trato igualitario y equitativo para alcanzar dicho desarrollo. La justicia está relacionada con la idea de rectitud dentro del entorno social (Stammler, 1958), también pudiera concebirse como la igualdad del derecho para todos (González de la Vega, 2005). La justicia se considera un derecho universal e individual; la concatenación de los intereses personales con los colectivos para establecer un balance o armonía entre ellos y la seguridad de los ciudadanos (Pound, 1951). Asimismo, se relaciona con un orden, con una posición del ser humano dentro de una esfera de interrelaciones personales y en coexistencia. 


\section{Justicia procedimental}

Las aproximaciones al estudio de la justicia en psicología se han dado desde diferentes lentes y niveles analíticos. En el área de psicología jurídica, se ha estudiado la justicia distributiva y la procedimental. La distributiva se refiere a la percepción de la equidad de los resultados o las sentencias en los juicios o procedimientos ante autoridades. La procedimental representa el sentimiento de imparcialidad o equidad que las personas perciben durante su participación en un procedimiento judicial. Algunas investigaciones han encontrado que a diferencia de la justicia distributiva, la justicia procedimental produce efectos más positivos desde el punto de vista de bienestar psicológico (Tyler \& Blander, 2000). La justicia procedimental pudiera considerarse, incluso, como el establecimiento de procesos imparciales o equitativos para que el resultado pueda considerarse como justo (Tyler, 2006). El término proviene del ámbito legal y hace referencia al "control ejercido por los afectados para resolver los casos jurídicos en el juzgado" (Tyler, 2006, p. 115).

En la actualidad una de las posturas con más influencia es la desarrollada por el grupo liderado por Tyler y Bies (1990). Según los autores, en nuestros encuentros diarios con las autoridades encargadas de distribuir las sanciones positivas o negativas, prestamos una especial atención a cómo se toman las decisiones y qué trato recibimos. En la medida en que juzguemos estos aspectos positivamente nos sentiremos más identificados con dichas autoridades y, por tanto, nos mostraremos más leales y dispuestos a obedecerlas. De acuerdo con esta perspectiva, la dimensión de la justicia procedimental determina de forma más intensa nuestra percepción de legitimidad, hasta el punto de llegar a aceptar resultados desfavorables siempre y cuando concluyamos que se derivan de la aplicación de procedimientos justos (Jost \& Major, 2001).

En este sentido, la justicia procedimental promueve la aceptación y el cumplimiento de las decisiones de las autoridades. Además, la gente que es tratada con justicia tiene más probabilidades de cooperar o cumplir con las disposiciones y evitar involucrarse en conducta antisocial. La justicia procedimental afecta las actitudes de las personas, específicamente, construye confianza interpersonal y en las autoridades; es más probable que las personas cooperen con aquellos a quienes tienen confianza (Lind, 1997). Un aspecto importante para las autoridades es que la gente responda bien a las evaluaciones de justicia, aun cuando el resultado no le favorezca (Tyler \& Huo, 2002).

La justicia procedimental (incluyendo transparencia, imparcialidad y respeto a la dignidad humana) proporciona legitimidad a las autoridades (Tyler, 2003). Dicha percepción de legitimidad de las autoridades origina que la población cumpla las disposiciones (Tyler, 2006). En el caso contrario es más probable que las personas desobedezcan y no cumplan con la ley en general. Igualmente, la justicia procedimental ayuda al seguimiento voluntario de las normas (Maiese, 2004). 
Fagan y Tyler (2005) exploraron las conexiones entre atribuciones de justicia procedimental, socialización legal y percepción de los adolescentes de tal justicia en el marco de sus interacciones con los actores legales y encontraron que era en el escenario de interacción donde se formaban las representaciones de los adolescentes sobre la legitimidad de la ley y sus instituciones. Además, una mejor percepción de la legitimidad del sistema legal estaba asociada con un bajo reporte de delincuencia juvenil. Estos autores sugieren una conexión entre las percepciones de justicia procedimental de los niños, el desarrollo de las concepciones de legitimidad del sistema y las conductas delictivas.

Las experiencias de los niños y jóvenes con el sistema legal repercuten en sus percepciones y respeto; existe una relación entre la percepción de su tratamiento en el gobierno y sus juicios de legitimidad de las autoridades con su conducta ilegal (Weisz, Wingrove, \& FaithSlaker, 2007). La legitimidad de la ley es la dinámica central del proceso de socialización de los niños. La investigación en el área ha encontrado que la gente adquiere puntos de vista sobre la legitimidad de las autoridades, que dicha apreciación forma su conducta, y se deriva de las interacciones interpersonales y la experiencia. Este proceso es identificado como parte del desarrollo de la adolescencia (Tyler \& Huo, 2002). Lo que los adolescentes ven y experimentan en la interacción con la policía y otros actores legales conforma su percepción sobre los individuos y la sociedad, esas experiencias influencian el desarrollo de sus nociones de ley, reglas, acuerdos entre los miembros de la sociedad, y la misma legitimidad de las autoridades.

La investigación con adultos sugiere que su experiencia con la ley contribuye a la evaluación de su legitimidad. Un tratamiento justo permite a la gente atribuirles legalidad a los aparatos de justicia y crear una serie de obligaciones para conformar sus normas. Un procedimiento justo reduce los sentimientos de enojo que llevan a romper las reglas. En otros términos, un tratamiento justo desarrolla ataduras y apego a la ley y a las normas sociales (Tyler \& Huo, 2002). Por lo tanto, el objetivo de esta investigación es analizar la relación entre la justicia procedimental, la aceptación de la sentencia y el tratamiento psicológico de los adolescentes en conflicto con la ley.

\section{Método}

\section{Participantes}

El instrumento se aplicó en una muestra total de 92 menores infractores, 50 de ellos se encontraban en internamiento en el Instituto de Tratamiento y de Aplicación de Medidas para Adolescentes (ITAMA) del Centro "La Victoria" con domicilio en Ejido La Victoria, Hermosillo, Sonora, quienes tenían entre 14 y 19 años de edad. La tabla 1 y 2 presentan las características demográficas de la muestra. 
Tabla 1

Frecuencias de las variables demográficas de los jóvenes en conflicto con la ley, tanto en internamiento como en externamiento

\begin{tabular}{llll}
\hline Variable & & Frecuencia & Total \\
\hline Sexo & Femenino & 3 & \\
& Masculino & 89 & 92 \\
Estado Civil & Soltero & 90 & \\
& Unión libre & 2 & 92 \\
Trabajas o has trabajado & No & 16 & 92 \\
& Sí & 76 & 92 \\
\hline
\end{tabular}

Igualmente, se consideraron 42 adolescentes que asistían al Centro de Tratamiento en
Externamiento cuyas edades fluctuaban de los 13 a los 19 años.

Tabla 2

Medias y desviaciones estándar de las variables demográficas de los menores en conflicto con la ley, tanto en internamiento como en externamiento

\begin{tabular}{lrrrrr}
\hline \multicolumn{1}{c}{ Variable } & N & Media & D. E. & Mínimo & Máximo \\
\hline Edad & 92 & 15 & 1.32 & 13 & 19 \\
Último grado terminado & 92 & 7 & 2 & 1 & 11 \\
Detenido por la policía & 92 & 4.47 & 5.63 & 0 & 30 \\
Edad del papá & 92 & 44 & 13 & 29 & 63 \\
Edad de la mamá & 92 & 39 & 9 & 30 & 56 \\
Ingreso mensual del padre & 92 & 6,800 & 4,998 & 1,500 & 30,000 \\
Ingreso mensual de la madre & 92 & 2,592 & 2,923 & 0 & 12,000 \\
\hline
\end{tabular}




\section{Instrumento}

Se aplicó una batería de pruebas consistente en tres escalas: la de percepción del tratamiento fue elaborada expresamente para esta investigación, las otras dos escalas son parte del estudio de Tyler (2006), las últimas dos fueron traducidas al español y piloteadas en una muestra de estudiantes mexicanos. Además, se agregaron variables demográficas como edad, sexo, estado civil, escolaridad, problemas de salud y de conducta. Así como, datos demográficos de los padres como: edad, estado civil, ocupación, número de hijos e ingreso mensual.

Percepción de trato de la policía. Once reactivos de Tyler (2006) conformaron esta escala encargada de medir cómo percibían los menores el trato recibido de la policía en general y en lo personal, con base en una escala del 0 al 10 en donde el 0 significaba nada de acuerdo y el 10 muy de acuerdo. Los participantes respondieron si el trato de la policía era justo o si se sentían satisfechos con él. Se obtuvo un alfa de Cronbach de .78 para esta escala.

Percepción del trato del juez. Doce reactivos fueron utilizados de la escala de Tyler (2006) para medir la apreciación que tienen los sujetos acerca del procedimiento judicial, desde que fueron detenidos hasta la fecha en que el juez les dictó sentencia. Los participantes respondieron en una escala del 0 al 10, es decir, desde qué tan injusta a qué tan justa fue su percepción acerca del tratamiento que recibieron durante el procedimiento. El alfa reportada para esta escala fue de .85 .
Percepción del tratamiento psicológico. Esta escala fue elaborada especialmente para el presente estudio, mide la percepción que tienen los sujetos acerca del tratamiento psicológico que reciben, tanto en internamiento como en externamiento del ITAMA. Los jóvenes reportaron su percepción mediante una escala tipo Likert de 5 puntos, donde las opciones fueron $0=$ nunca, $1=$ pocas veces, $2=$ algunas veces, $3=$ muchas veces y $4=$ siempre. Se produjo un alfa de .64 .

\section{Procedimiento}

Para el caso de los menores internos se solicitó al director del Centro de Internamiento "Granja Lic. Marco Antonio Salazar Siqueiros" de ITAMA el permiso para la aplicación del instrumento. Los adolescentes respondieron el cuestionario en un aula del Centro de Internamiento, en 5 grupos de 10 sujetos cada uno. Al coordinador del Centro de Tratamiento en Externamiento se le pidió autorización para la aplicación del cuestionario a los jóvenes que asistían a terapia psicológica en dicho Centro. Un total de 42 jóvenes contestaron de forma individual el instrumento al momento de entrar a consulta en psicología. Una psicóloga estuvo aclarando sus dudas y proporcionando las instrucciones. Los jóvenes fueron informados de que su participación sería totalmente voluntaria y que podían dejar de contestar el cuestionario en cualquier momento. De la misma manera se les aseguró total confidencialidad de la información que proporcionaron. 


\section{Análisis de datos}

Los datos fueron analizados en el programa estadístico SPSS (2007), por medio del cual se realizaron estadísticas univariadas, y se obtuvieron las frecuencias de las variables discretas, medias y desviaciones estándar para las variables continuas. Así mismo, se produjeron alfas de Cronbach de las escalas utilizadas. Posteriormente, se elaboraron parcelas con los reactivos de las escalas de percepción del trato de los jueces y la policía. Las parcelas se forman con el promedio de un número de reactivos de cada escala tomados al azar. Las parcelas se justifican en el modelamiento estructural cuando los números de factores e indicadores son elevados y pueden dificultar la estimación de las relaciones entre variables (Little, Cunningham, Shahar \& Widaman, 2002). De esta manera resultaron 3 parcelas de la escala de percepción del trato de los jueces y 3 de los policías. Además, se probó un modelo de ecuaciones estructurales; las relaciones entre las variables fueron hipotetizadas en el modelo mostrado en la figura 1. Las parcelas fueron indicadores de cada uno de los factores de primer orden señalados como círculos en la figura 1: percepción del juez y percepción del policía otras variables latentes serían la aceptación

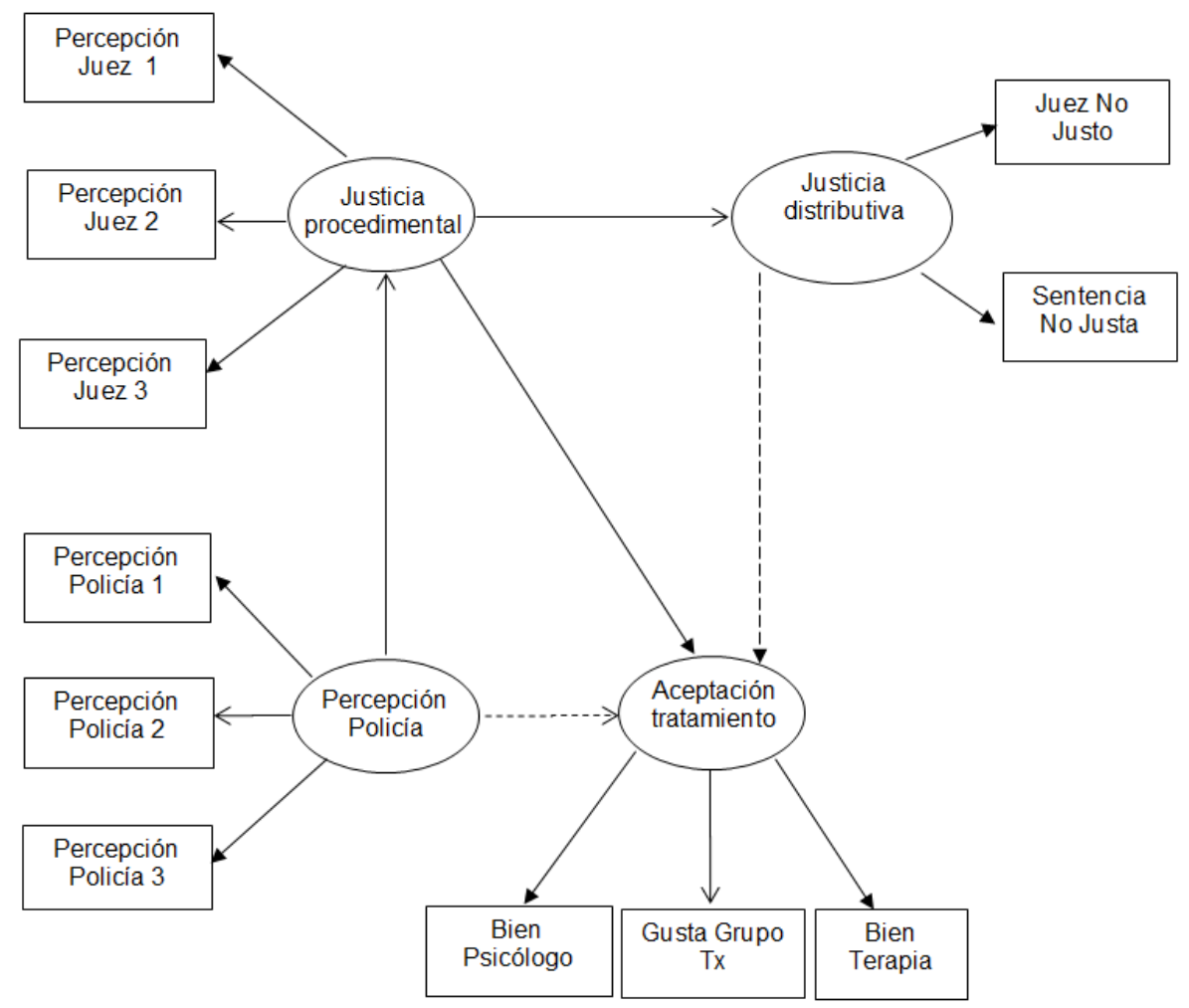

Figura 1. Modelo hipotético de relaciones entre las variables del estudio. 
al tratamiento conformada por aceptación del psicólogo, de la terapia y del grupo de terapia y la aceptación del procedimiento con percepción de la sentencia y juez justo.

Varios indicadores de bondad de ajuste fueron considerados en el modelo. La bondad de ajuste nos muestra la pertinencia de la teoría y las relaciones entre las variables establecidas en el modelo. Indicadores prácticos y estadísticos fueron utilizados para medir la bondad de ajuste (Ullman, 2006). Los indicadores estadísticos incluyen la $X^{2}$, que deberá tener un valor bajo y no significativo $(p>.05)$. Sin embargo, como éstos son sensibles al tamaño de la muestra, los indicadores prácticos son considerados (Bentler, 2006). Los indicadores prácticos derivan de la $X^{2}$ y controlan los efectos del tamaño de la muestra en los niveles de significancia. Los indicadores prácticos utilizados fueron Bentler-Bonnet Normed Fix Index (BBNFI), Comparative Fix Index (CFI), y Bentler-Bonnet Non-Normed Fix Index (BBNNFI). Los cuáles deberán tener un valor mayor a .90 (Bentler, 2006); el cuadrado del error de medias (RMSEA) requiere un valor menor de .08 (Browne \& Cudeck, 1993).

\section{Resultados}

Análisis univariados y confiabilidad de las escalas. La tabla 3 muestra los análisis de confiabilidad, medias, desviaciones estándar, puntajes mínimos y máximos que produjeron las variables con las cuales se constituyeron las escalas del estudio; las alfas de Cronbach estuvieron por encima de .60, por lo que se consideran aceptables. Para la escala de Percepción del tratamiento psicológico, las medias puntuaron desde 2.94 a 3.84 (en un rango del 0 al 4 de posibles respuestas), es decir, los adolescentes que reciben tratamiento tanto en internamiento como en externamiento perciben de manera positiva la atención psicológica del Instituto. En la escala referente a la satisfacción del trato del policía de 11 reactivos 9 obtuvieron una media de entre 2.71 a 4.69 (en un rango del 0 al 10 de posibles respuestas), esto indica que los jóvenes en conflicto con la ley perciben a la corporación policiaca como deshonestos e injustos en su trato, ya que no les explican sus derechos al detenerlos y consideran que no resuelven los problemas de los ciudadanos de manera adecuada, por lo tanto, no se sienten orgullosos ni tienen respeto por los oficiales de policía. En cambio, para la escala Percepción del trato del juez los menores respondieron con puntuaciones que van de 6.10 a 7.73 (rango del 0 al 10), lo cual significa que perciben a los jueces como justos, honestos, respetuosos de los derechos básicos de los ciudadanos y sienten satisfacción en el trato recibido por éstos. 
Tabla 3

Alfas de las escalas que componen el cuestionario para la muestra de la población estudiada

\begin{tabular}{|c|c|c|c|c|c|c|}
\hline Escala/ Reactivos & $\mathrm{n}$ & $x$ & D.E. & Min & Max & Alfa \\
\hline Percepción del tratamiento psicológico & & & & & & .64 \\
\hline $\begin{array}{l}\text { La psicóloga me entiende lo que quiero } \\
\text { comunicarle. }\end{array}$ & 90 & 3.64 & .90 & 0 & 4 & \\
\hline Me siento bien con la psicóloga que me tocó. & 90 & 3.84 & .65 & 0 & 4 & \\
\hline Siento que me ayuda ir con la psicóloga. & 90 & 3.64 & .81 & 0 & 4 & \\
\hline Me gusta mucho ir a los grupos. & 86 & 2.94 & 1.40 & 0 & 4 & \\
\hline Me la paso bien en los grupos. & 86 & 3.04 & 1.42 & 0 & 4 & \\
\hline Percepción del trato de la policía & & & & & & .78 \\
\hline Tengo un gran respeto a la policía. & 92 & 4.69 & 3.75 & 0 & 10 & \\
\hline Los oficiales de policía son honestos. & 92 & 2.71 & 3.22 & 0 & 10 & \\
\hline Me siento orgulloso de la policía. & 92 & 3.25 & 3.30 & 0 & 10 & \\
\hline $\begin{array}{l}\text { Cuando me detienen me explican mis } \\
\text { derechos. }\end{array}$ & 92 & 2.84 & 3.82 & 0 & 10 & \\
\hline $\begin{array}{l}\text { Se le debe permitir a la policía detener } \\
\text { a las personas que son sospechosas de } \\
\text { delitos graves hasta que obtengan suficiente } \\
\text { evidencia para acusarlos oficialmente. }\end{array}$ & 92 & 8.19 & 3.21 & 0 & 10 & \\
\hline $\begin{array}{l}\text { Cómo sientes el trato de la policía cuando } \\
\text { tienes problemas en la calle. }\end{array}$ & 92 & 3.40 & 3.41 & 0 & 10 & \\
\hline Qué tan justos sentiste los resultados. & 92 & 4.42 & 3.62 & 0 & 10 & \\
\hline $\begin{array}{l}\text { Qué tan satisfecho estás con la policía cuando } \\
\text { resuelve los problemas de la ciudad y la ayuda } \\
\text { que da a la gente. }\end{array}$ & 92 & 6.55 & 3.34 & 0 & 10 & \\
\hline $\begin{array}{l}\text { La policía trata a todos equitativamente o hace } \\
\text { diferencias a favor de algunos. }\end{array}$ & 92 & 3.73 & 3.57 & 0 & 10 & \\
\hline $\begin{array}{l}\text { La gente como tú recibe el mismo trato de la } \\
\text { policía o hay diferencias. }\end{array}$ & 92 & 4.19 & 3.16 & 0 & 10 & \\
\hline $\begin{array}{l}\text { Cuando te ha detenido la policía ha manejado } \\
\text { la situación satisfactoriamente. }\end{array}$ & 92 & 4.46 & 3.87 & 0 & 10 & \\
\hline Percepcion del trato del juez & & & & & & .86 \\
\hline $\begin{array}{l}\text { Te has sentido satisfecho con el trato que has } \\
\text { recibido del juez. }\end{array}$ & 92 & 7.25 & 3.53 & 0 & 10 & \\
\hline $\begin{array}{l}\text { Qué tan satisfecho te has sentido con el poder } \\
\text { judicial cuando oyes de algún caso. }\end{array}$ & 92 & 6.10 & 3.03 & 0 & 10 & \\
\hline Piensas que los jueces son justos. & 92 & 7.22 & 3.05 & 0 & 10 & \\
\hline $\begin{array}{l}\text { Piensas que los jueces resuelven los casos } \\
\text { con justicia. }\end{array}$ & 92 & 7.54 & 2.90 & 0 & 10 & \\
\hline
\end{tabular}


Tabla 3 (Cont.)

Los jueces tratan a todos equitativamente o hacen diferencias a favor de algunos.

La gente de tu edad recibe el mismo trato de los jueces o crees que hay diferencias.

Los jueces garantizan para todos un juicio justo.

Los jueces son demasiado blandos con los criminales.

Los derechos básicos de los ciudadanos son bien protegidos por los jueces.

En general, los jueces son honestos. Las decisiones de los jueces son siempre justas.

$\begin{array}{lllll}92 & 5.93 & 3.43 & 0 & 10 \\ 92 & 5.79 & 3.39 & 0 & 10 \\ 92 & 7.38 & 3.35 & 0 & 10 \\ 92 & 4.54 & 3.86 & 0 & 10 \\ 92 & 7.73 & 2.97 & 0 & 10 \\ 92 & 7.11 & 3.32 & 0 & 10 \\ 92 & 6.36 & 3.60 & 0 & 10\end{array}$

La figura 2 muestra los resultados del modelo estructural, los resultados del modelo de medición son representados por los círculos así como los indicadores por rectángulos. Los factores de percepción surgieron de manera coherente de las relaciones entre los indicadores, es decir, de las tres parcelas producidas a partir de los reactivos correspondientes a cada factor. Los pesos factoriales altos $\mathrm{y}$ significativos $(p$ $<$.05) muestran que los cuatro factores poseen validez de constructo convergente. Estas cargas factoriales fueron para la Percepción del juez $.78, .75$ y .66 para la primera, segunda y tercera parcela, respectivamente. Para el caso de la Percepción de la policía, los pesos factoriales fueron de $.76, .83$ y .66 en la primera, segunda y tercera parcela, correspondientemente. En la Percepción del procedimiento judicial las cargas factoriales fueron de .75 para el "juez no es justo" y de .66 para "la sentencia no es justa". Para la Aceptación del tratamiento los pesos factoriales fueron .39 para "me siento bien con el psicólogo", .72 para "me gusta ir a los grupos" y .84 "siento que me ayuda la terapia".

En el modelo estructural el factor Percepción del trato del policía $(C E=.67)$ tuvo efecto directo en la Percepción del trato del juez, el cual afecta la Aceptación del tratamiento psicológico ( $C E$ $=.42$ ), la Percepción del juez afectó de manera positiva a la Percepción del procedimiento judicial $(C E=.77)$.

Los indicadores de bondad de ajuste del modelo resultante fueron $X^{2}=49.37$ (39 g.l.) a la cual se asocia una $p=0.12$, los valores de los indicadores prácticos de bondad de ajuste fueron Bentler-Bonett de ajuste no-normado $B B N F I=0.95$, e índice de ajuste comparativo $C F I=0.96$, resultando mayor a 0.90 como se 


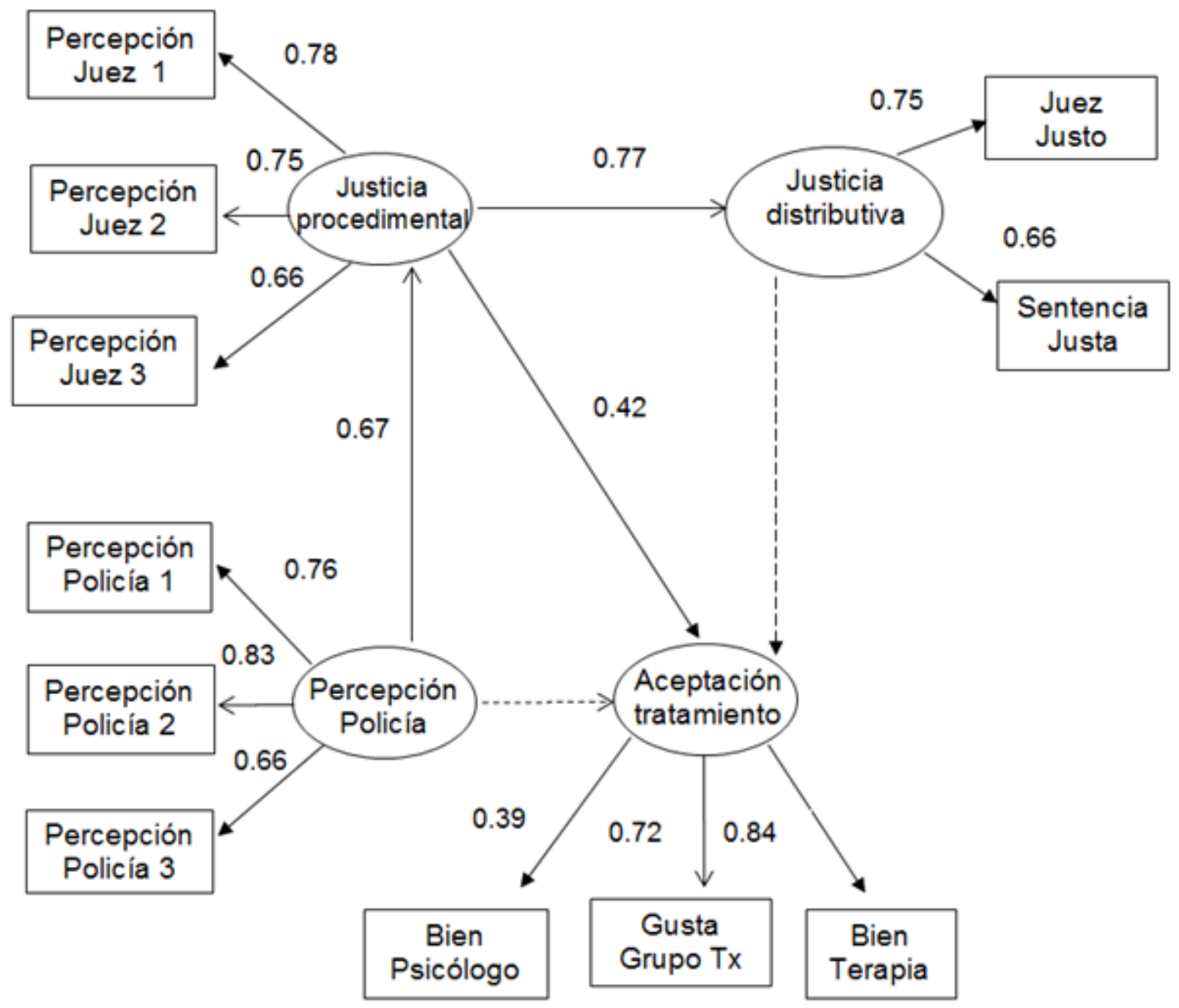

Figura 2. Resultados del modelo de la percepción de los adolescentes en conflicto con la Ley del procedimiento judicial y del tratamiento psicológico que reciben en internamiento o en externamiento. $R^{2}=.35 . X^{2}=49.37$ (39 g.l.); $p=.12 ; B B N F I=.95$; $C F I=.96 ;$ RMSEA $=.05$.

esperaba y con un $R M S E A=0.05$, menor a 0.08 . La $R^{2}$ del modelo fue de 0.35 , lo cual significa que el modelo predice el $35 \%$ de la varianza de la aceptación del tratamiento. Estos valores indican que el modelo se ajusta a los datos obtenidos (Bentler, 1993).

\section{Discusión}

Los resultados nos mostraron que la percepción que tienen los menores infractores sobre el trato que reciben de los jueces está predicha por el comportamiento que apreciaron 
de los policías. La percepción de la actuación de los jueces afectó la aceptación del procedimiento judicial y el tratamiento psicológico por parte de los jóvenes. Esto nos quiere decir que si los adolescentes sienten que son bien tratados por los jueces van a aceptar mejor el tratamiento psicológico y la sentencia que les aplican.

Las políticas públicas que emanaron de las reformas Constitucionales para la protección integral del menor y la protección de sus derechos parece que no se están cumpliendo. Ya que los menores en tratamiento en ITAMA, ya sea en internamiento o en externamiento, señalan a los oficiales de policía de manera negativa, es decir, como deshonestos e injustos en su trato. Los policías deberán de cambiar sus actitudes y conducta para estar acorde con estas políticas públicas emanadas de la reforma Constitucional. La protección de los derechos de los menores deberá de establecerse desde el primer contacto con las autoridades. Los resultados del estudio nos indican que si falla esta primera interacción con las autoridades puede fallar la integración del menor en el proceso y en la sociedad.

Además, el tratamiento psicológico lo reciben con aceptación, ya que refieren sentir que les ayuda ir a terapia psicológica y que asisten a la terapia grupal con gusto. Por lo tanto, la percepción que los jóvenes tengan del juez influye en la aceptación del tratamiento psicológico, es decir, si encuentran que el juez los trató con respeto y defiende los derechos básicos de los ciudadanos, entonces aceptarán la terapia psicológica, asistiendo con agrado a las sesiones y reportando sensación de mejoría; igualmente, la percepción de los jóvenes de un Juez justo les permitirá considerar justa la sentencia que han recibido en proporción al delito que han cometido.

Fagan y Tyler (2005) nos sugieren una relación entre la justicia procedimental y las conductas delictivas en los jóvenes, los datos nos muestran que si se percibe un trato justo por parte del juez aceptan más fácilmente la sentencia y el tratamiento; esto pudiera ayudar a la disminución de la reincidencia. E1 tratamiento justo le proporciona legalidad a las autoridades y crea en los ciudadanos una serie de obligaciones para el seguimiento de normas. Por consiguiente, un proceso equitativo pudiera desarrollar apego a la ley en los adolescentes, ya que perciben que fueron tratados con justicia (Tyler \& Huo, 2002).

Las implicaciones del estudio tendrían relación con la implementación de las políticas públicas emanadas de las leyes. Es muy importante considerar que si los principios fundamentales de la atención al menor, como es el trato justo, no se aplican, no se va a poder incidir en la reducción de la reincidencia de los adolescentes. Los policías tienen que ser entrenados en el tratamiento de los menores. Tienen que conocer los derechos de los adolescentes y las garantías que establece la Constitución para ellos. Parece que el problema principal que presenta la atención y tratamiento de los menores en conflicto con la ley penal es que no se están aplicando los principios 
fundamentales que establecen la Constitución y las políticas públicas que se derivan de ellos. Por lo tanto, se recomienda que los derechos de los adolescentes sean garantizados y que las personas que los vayan a atender tengan un entrenamiento especializado, desde los policías, ministerios públicos, jueces, abogados, y psicólogos.

La limitación que se presentó para este estudio ha sido el tamaño de la muestra, específicamente en lo referente a los jóvenes que reciben su tratamiento en externamiento, ya que éstos son citados en las instalaciones de la Institución y pueden faltar a la sesión. No se les indica cuando ni cuánto tiempo tienen para cumplir el tratamiento en externamiento, solo se les dicta la duración sentencia, por lo tanto, ésta empieza a contar a partir de la primera sesión a la que asisten a tratamiento psicológico.

\section{Referencias}

Bentler, P. M. (2006). EQS 6 Structural Equations Program Manual. Encino, CA: Multivariate Software Inc.

Browne, M. W., \& Cudeck, R. (1993). Alternative ways of assessing model fit. En K. A. Bollen \& J. S. Long (Eds.), Testing structural equation models (pp. 136-162). Thousand Oaks, CA: Sage.

Constitución Política Mexicana. (2013). Recuperado de http://www.diputados.gob. mx/LeyesBiblio/pdf/1.pdf.
Diario Oficial de la Federación. (2005). Recuperado de http://www.diputados. gob.mx/LeyesBiblio/ref/dof/CPEUM_ ref_165_12dic05_ima.pdf.

Fagan, J., \& Tyler, T. R. (2005). Legal Socialization of Children and Adolescents. Social Justice Research, 18(3), 217-241.

González de la Vega, R. (2005). Justicia e ideología. México DF: Universidad Autónoma de México, Instituto de Investigaciones Jurídicas.

Instituto Nacional de Estadística, Geografía e Informática (INEGI). (2010). México: Anuario estadístico del Estado de Sonora.

Instituto Nacional de Estadística, Geografía e Informática (INEGI). (2011). México: Anuario de Estadísticas por Entidad Federativa.

Jiménez O. R. (2011). Violencia Social y Vulnerabilidad Juvenil. Instituto Ciudadano de Estudios Sobre la Inseguridad, A. C. Recuperado de http://www.icesi.org.mx/ publicaciones/articulos/2002/violencia_ social_y_vulnerabilidad_juvenil.asp.

Jost, J. T., \& Major, B. (2001). The Psychology of Legitimacy: Emerging Perspectives on Ideology, Justice and Intergroup Relations. (2a ed.). Londres: Cambridge University Press.

Lind, E. A. (1997). Litigation and claiming in organizations: Antisocial behavior or quest for justice? En R. A. Giacalone \& J. Greenberg (Eds.), Antisocial behavior in 
organizations (pp. 150-171). Thousand Oaks, CA: Sage.

Little, T., Cunningham, W., Shahar, G., \& Widaman, K. (2002). To parcel or not parcel: Exploring the question, weighing the merits. Structural Equation Modeling: A Multidisciplinary Journal, 9, 151-173.

Maiese, M. (2004). Procedural Justice. Beyond Intractability. En G. Burgess \& H. Burgess (Eds.), Conflict Information Consortium, University of Colorado, Boulder. Recuperado de: <http://www.beyondintractability.org/biessay/procedural-justice $>$.

Perelman, C. (1964). De la Justicia. México DF: Universidad Autónoma de México, Instituto de Investigaciones Jurídicas.

Pound, R. (1951). The need of a sociological jurisprudence. Justice According to Law. New Haven, CT: Yale University Press.

Manual del usuario del sistema básico de IBM SPSS Statistics 20. (2011). Copyright IBM Corporation 1989, 2011. Recuperado de ftp://public.dhe.ibm.com/software/analytics/ spss/documentation/statistics/20.0/es/client/ Manuals/IBM_SPSS_Statistics_Core_ System_Users_Guide.pdf.

Stammler, R. (1958). La esencia del derecho y la ciencia del derecho. Madrid: Espasa-Calpe.

Tyler, T. R. (2003). Procedural justice, legitimacy, and the effective rule of law. En M. H. Tonry (Ed.), Crime and justice: A review of research (Vol. 30, pp. 431-505). Chicago: University of Chicago Press.
Tyler, T. R. (2006). Perception of adolescents about the judicial procedure and treatment in detention and their effect on social rehabilitation. Princenton: Princeton University Press.

Tyler, T. R., \& Bies, R. J. (1990). Beyond formal procedures: The interpersonal context of procedural justice. In J. Carroll (Ed.), Applied social psychology and organizational settings, (pp.77-98). Hillsdale, NJ: Erlbaum Associates.

Tyler, T. R., \& Blader, S. L. (2000). Cooperation in groups: Procedural justice, social identity and behavioral engagement. Philadelphia, PA: Psychology Press.

Tyler, T. R., \& Huo, Y. J. (2002). Trust in the law: Encouraging public cooperation with the police and courts. New York: Russell Sage Foundation.

Tyler, T. R., \& Mentovich, A. (2011). Mechanisms of Legal Effect: Theories of Procedural Justice. A methods monograph. New York: Robert Wood Johnson Foundation.

Ullman, J. B. (2006). Structural equation modeling: Reviewing the basics and moving forward. Journal of Personality Assessment, $87,35-50$.

Weisz, V., Wingrove, T., \& Faith-Slaker, A. (2007). Children and Procedural Justice. Court Review. The Journal of the American Judges Association, 44, 216. Recuperado de http:// digitalcommons.unl.edu/ajacourtreview/216. 